\title{
Chains of benzenes with lithium-atom adsorption: Vibrations and spontaneous symmetry breaking
}

\author{
Yenni P. Ortiz ${ }^{\mathrm{a}}$, Thomas Stegmann ${ }^{\mathrm{a}}$, Douglas J. Klein ${ }^{\mathrm{b}}$, Thomas H. Seligman ${ }^{\mathrm{a}, \mathrm{c}}$ \\ ${ }^{a}$ Instituto de Ciencias Físicas, Universidad Nacional Autónoma de México, Avenida Universidad s/n, 62210 Cuernavaca, México \\ ${ }^{b}$ Texas \& M University at Galveston, TX 77551, USA \\ ${ }^{c}$ Centro Internacional de Ciencias, 62210 Cuernavaca, México
}

\section{N Abstract}

We study effects of different configurations of adsorbates on the vibrational modes as well as symmetries of polyacenes and poly$p$-phenylenes focusing on lithium atom adsorption. We found that the spectra of the vibrational modes distinguish the different configurations. For more regular adsorption schemes the lowest states are bending and torsion modes of the skeleton, which are essentially followed by the adsorbate. On poly-p-phenylenes we found that lithium adsorption reduces and often eliminates the torsion between rings thus increasing symmetry. There is spontaneous symmetry breaking in poly-p-phenylenes due to double adsorption of lithium atoms on alternating rings.

Keywords: conjugated carbon structures, spontaneous symmetry breaking

\section{Introduction}

Adsorption of lithium atoms and other alkali metals to conjugated carbon systems has shown the possibility of strong deformations which may occur as spontaneous symmetry breaking [1, 2, 3, 4, 5]. Polyacenes have served as paradigmatic examples [1, 2, 5] but similar effects have previously been discussed in other aromatic molecules [3, 5]. The question as to what extent such adsorption can be detected leads to a comparison between the vibrational spectra of the corresponding molecules. One focus here is on polyacenes, in particular on anthracene for two reasons: First, it is in this context that interesting near-periodic structures are seen which are relevant to narrow graphenic nano-ribbons [4]; Second, the matter of spontaneous symmetry breaking is quite clear in these structures, as we shall see. Further, another type of benzenoid species is studied, namely the poly-p-phenylenes (PPP) we find that adsorption here increases certain (distortive) similarities to polyacenes, in that torsion present in the naked PPP is suppressed. Notably PPPs are less chemically reactive (and so much easier to handle) as compared to polyacenes. Also their potential for branching allows a richer spectrum of future extension of the present work.

The question arises as to how such deformations affect the stiffness of the molecules and to what extent low-lying modes couple movements of the underlying chains and movements of adsorbates. We study Li adsorption, manifesting interesting effects: spontaneous symmetry breaking and fairly large charge transfers. The extension to linear PPPs is very suggestive because they represent the narrowest "armchair" graphene boundary [6, 7]. One great difference is that (neutral) PPPs are not flat and thus seem to be far from armchair edge nano-strips but interestingly we find that they flatten when subject to adsorption of lithium atoms.

We use DFT calculations to obtain the pertinent data from which we obtain the structure and further data that determine the vibrational modes. We then proceed to calculate the vibrational modes. Next we discuss the differences in the spectra and observe that with few exceptions the actual dynamics of low lying frequencies is much the same as that of the underlying spectrum of the simple (naked) polyacenes. The purpose is to show how things change with heavier and larger adsorbates.

The need to analyze vibrational modes of such systems naturally arises to enhance structural understanding. On one hand we would like to know if the spectra are sufficiently different to allow the identification of a given configuration and on the other hand, whether the structure of low lying modes indicates some decoupling of the movement of the naked molecule (skeleton) from the movement of the adsorbates. If indeed such a decoupling happens it would be important to know which modes are lower. We present this analysis in some detail for the case of anthracene, to find that the spectra are quite different; furthermore we find the usual scenario, the first few modes are vibrations of the skeleton which are essentially followed by the adsorbates, though exceptions occur. These results will lead us to a speculative discussion of the possibility to use such chains, most likely PPP's, with regularly spaced adsorbates as substrate for quantum register using the adsorbates as the string of qubits and the vibrations as extended qubits to mediate the information for control gates in the spirit of the Innsbruck quantum computer [8, 9]. There an ion chain in a casi 1-D trap serves as qubit chain and the collective motion of the ions to mediate information for control gates. We will not discuss the quantum optical necessities to implement such a scheme coupling the ions by molecular vibrations and the qubits by adsorbed $\mathrm{Li}$ atoms. Rather we simply point out that a possibly viable structure ex- 
ists. In particular we present some configurations of polyacenes and PPP's that fulfill the obvious criteria we need: Low lying largely decoupled modes of movement of the skeleton.

For PPPs torsion modes will also appear in the lower part of the spectra, and they similarly tend to decouple at low frequency from the other modes, this have been seen in other carbon systems [10]. Again there are exceptions. Nevertheless, the prevailing feature of decoupling of modes for the lowest states is interesting and may be relevant in various aspects not presently obvious.

We proceed as follows: First we discuss briefly the computational methods used. We then proceed to discuss anthracene in detail analyzing 14 configurations with zero up to four lithium atoms adsorbed. Some aspects relating to spontaneous symmetry breaking in infinite systems and their relation to the Peierls transition are commented as supplement. Finally we give some conclusions and an outlook.

\section{Computational methods}

The calculations for individual molecules were performed with the GAUSSIAN09 program codes [11]. Geometry optimizations were made with DFT [12] using hybrid functional B3LYP [13]. We have selected the 6-311g [14] basis sets for electronic structure calculations that use linear combinations of gaussian functions to form the orbitals. Additionally, we use the improvement of these basis sets by adding $d$ functions to carbon atoms.

To test whether polarization is important we rerun some of the calculations with two different basis sets that include such terms, 6-31g+(d) and 6-311++g(d,p). Also we used alternatively the functionals MPW1PW91 [15, 16, 17] and B3PW91 [13, 15, 18]. Those functionals have been used in carbons systems [19, 20] as well as in alkaline molecules [21] which have been reported to yield very accurate vibrational frequencies and IR intensities. No qualitatively difference could be seen in the results, both for the structure and the vibrational spectra, therefore we do not show the corresponding figures.

The frequency calculations were made with the same package, by determining the second derivatives of the energy with respect to the Cartesian nuclear coordinates and then transforming to mass-weighted coordinates [11]. We have used the options corresponding to the low frequency vibrational modes (opt=verytight, int=grid=ultrafine $)$ in the GAUSSIAN09 program.

\section{Results and discussion}

In general terms, we have found qualitative similar results with the three functionals, particularly all of them keep the same minimum vibrational state, except for the case of the configuration 11 that is shown in Figure 1. The results shown in this paper are those with the functional B3LYP.

\subsection{Anthracene $\mathcal{E}$ Nonacene}

Results for the vibrational modes of anthracene are shown in Figure 11. The different spectra are ordered with increasing numbers of adsorbates starting from zero up to three for the configurations our calculations show to be stable. In this figure and in all following vibrational spectra, we give just the first 15 lower energy modes. Tight doublets are indicated by the letter $\mathrm{D}$ to make sure that they can be identified. The actual movements for each configuration are shown in a set of short movies available in the complementary materials. These movies are given for the lowest three modes of all configurations shown in the figures with spectra, and they are identified as follows: $n_{1}-n_{2}-n_{3}$, where $n_{1}$ identifies the number of the figure in this article, $n_{2}$ the column in which the structure appears and $n_{3}$ the mode to which the clip belongs.

The first thing we notice in Figure 1, is that different numbers of adsorbates have different level densities. We can then focus on situations with the same number of adsorbates and try to see whether different configurations have markedly different spectra. This is indeed the case, and thus vibrational spectra which are qualitatively correct, will identify the structure. The next step is to see if the two, three and four adsorbate structures which have large deformation, i.e. the ones with one or two benzene rings with two sided adsorption have any characteristic difference, as compared to the other structures. Here we must first draw attention to a case not previously studied. In column 12 of Figure 1 we see a skeleton with large deformation with three Li adsorbates. The angle formed by the skeleton is actually even more pronounced then for the previously studied situation of column 4. Furthermore we can see in the corresponding movie clip, that the lowest vibrational mode is essentially only the $\mathrm{Li}$ at the apex performing a large oscillation. A check of the charges, using the natural-bond-orbital analysis, shows that the adsorbed $\mathrm{Li}$ are electropositive. The total transferred charge for the configurations of column 4 and 12 are roughly equal. In configuration 4 the $\mathrm{Li}$ at the apex and the $\mathrm{Li}$ inside the wedge transfer almost the same charge. In configuration 12 the $\mathrm{Li}$ at the apex transfers the same charge as the corresponding $\mathrm{Li}$ in configuration 4 , while the two Li inside the wedge transfer each almost the half of it. In the movement of the different configurations we see, not unexpectedly, that some modes involve essentially only movement of one or more adsorbates as seen best in the above example.

After having shown the more obvious effects of adsorption on vibrational spectra one thing emerged. There is a real chance for quasi-one-dimensional configurations which are also fairly flat if the distribution of the adsorbates is more or less uniform along the string. In this case the lowest modes mostly consist of an oscillation of the skeleton which is uniformly followed by the adsorbates, which then do not much change their position relative to the benzene ring to which they are adsorbed. To confirm our observations we look at selective situations of $\mathrm{Li}$ adsorption to nonacene. Figure 2 already shows such four configurations for nonacene. In view of the wide field of molecules we could extend this analysis to, a very hypothetical application may be considered to guide us. The Innsbruck group of R. Blatt devised a quantum computer based on a quasi-1-D ion trap as 


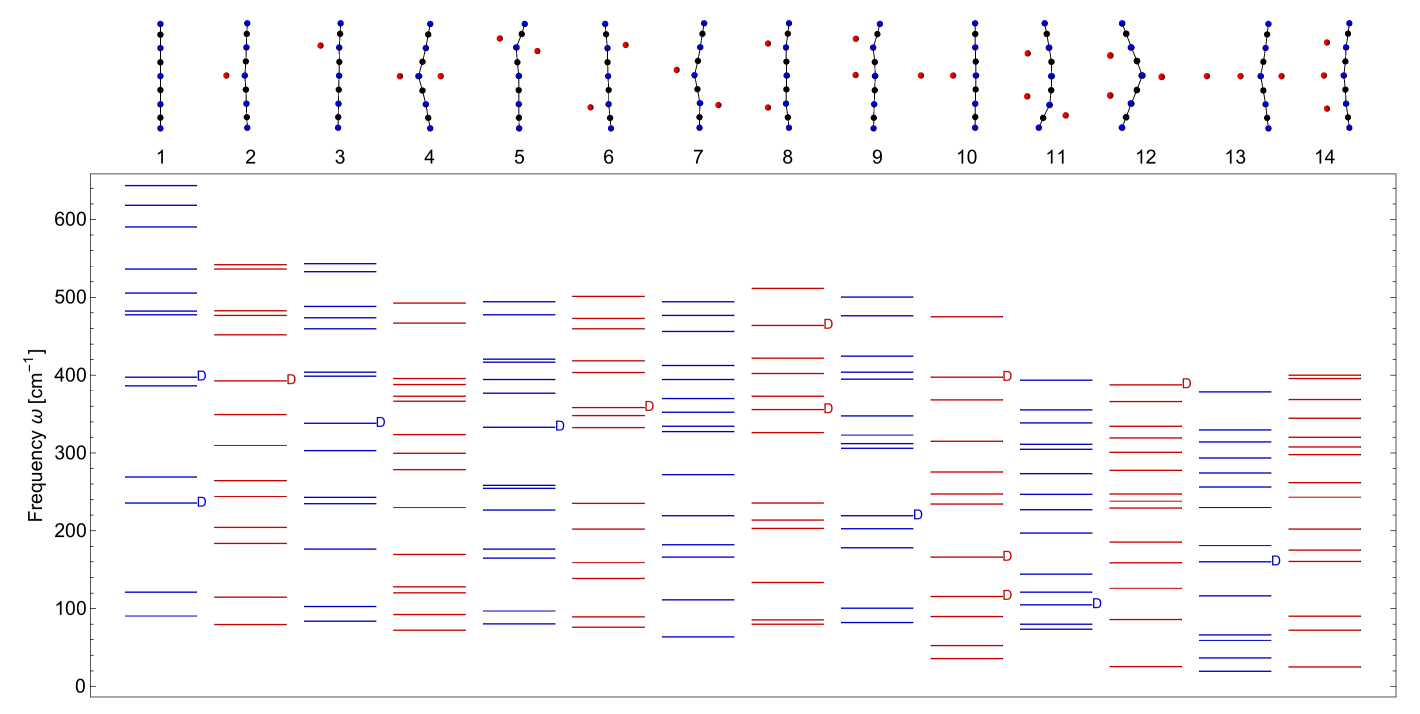

Figure 1: Vibrational spectra of anthracene and anthracene with Li adsorption. In this figure and in all following vibrational spectra, 15 modes are shown for each molecule. Tight doublets are indicated by the letter $\mathrm{D}$ to make sure that they can be identified.

mentioned in the introduction. We shall look at the behavior of longer chains but restrict our considerations to reasonably flat and symmetric configurations. Figure 2 already shows such configurations for nonacene. Again we see the lowest bending and torsional modes are of the type where the strings with $\mathrm{Li}$ adsorption just follow the movements of the naked skeletons, making them nice further examples we found in anthracene.

\section{2. poly-p-phenylenes}

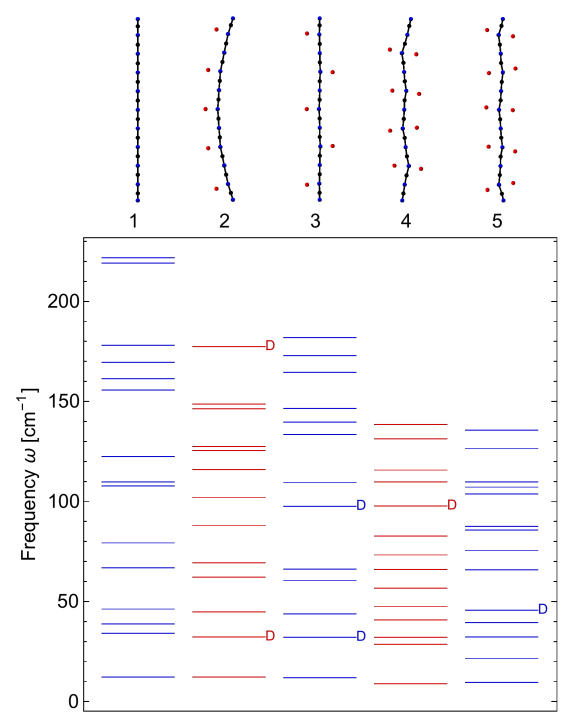

Figure 2: Vibrational spectra for nonacene and for some configurations of nonacene under $\mathrm{Li}$ adsorption.

To carry this theme a little further let us try to be a little more realistic to deal with species less reactive than the (higher) polyacenes, which tend to oxidize on exposure to ordinary atmospheric conditions. Indeed this reactivity rapidly increases with polyacene chain length - so that the outlook for long chains is (a)

(b)

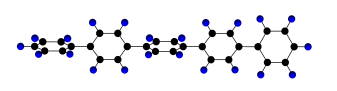

(c)
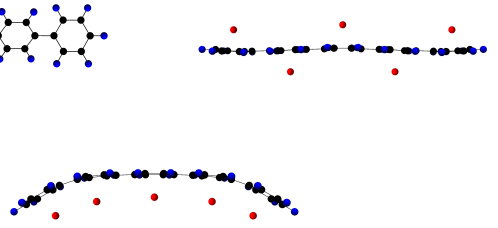

Figure 3: (a) Pentaphenyl, (b) Lateral view from adsorption of five lithium atoms alternating ring and side on pentaphenyl, $(c)$ Lateral view from adsorption of five lithium atoms on the same side on pentaphenyl

much better if we look at phenylenes. These are readily available for large branchings if so desired, though we shall stick to linear chains or PPPs for the present paper. An apparent disadvantage is the fact that the benzene rings often twist against one another, at any chain length. Yet in Figure 3 we see for pentaphenyl an interesting feature: both single and double adsorption seem to eliminate this torsion to a large degree, at least if the adsorbates cover the length of the PPP in an ordered manner. We can thus proceed to study vibrations in several configurations obtained from DFT calculations. In Figure 4 we show selected configurations of triphenyl under lithium adsorption. The different spectra are ordered with increasing numbers of adsorbates starting from zero up to three for the configurations our calculations show to be stable. The actual movements for each configuration are shown in a set of short movies available in the complementary materials. Yet the question remains if $\mathrm{Li}$ in partial ionization will provide an adequate two- level system and appropriate conditions can be established. This cannot be the subject of this work, as it would go deeply into quantum optics.

The adsorbed Li atoms are electropositive, and so should give up electrons to leave excess-electron-defected PPP. Then within a resonance-theoretic view it should be favorable for the excess- 


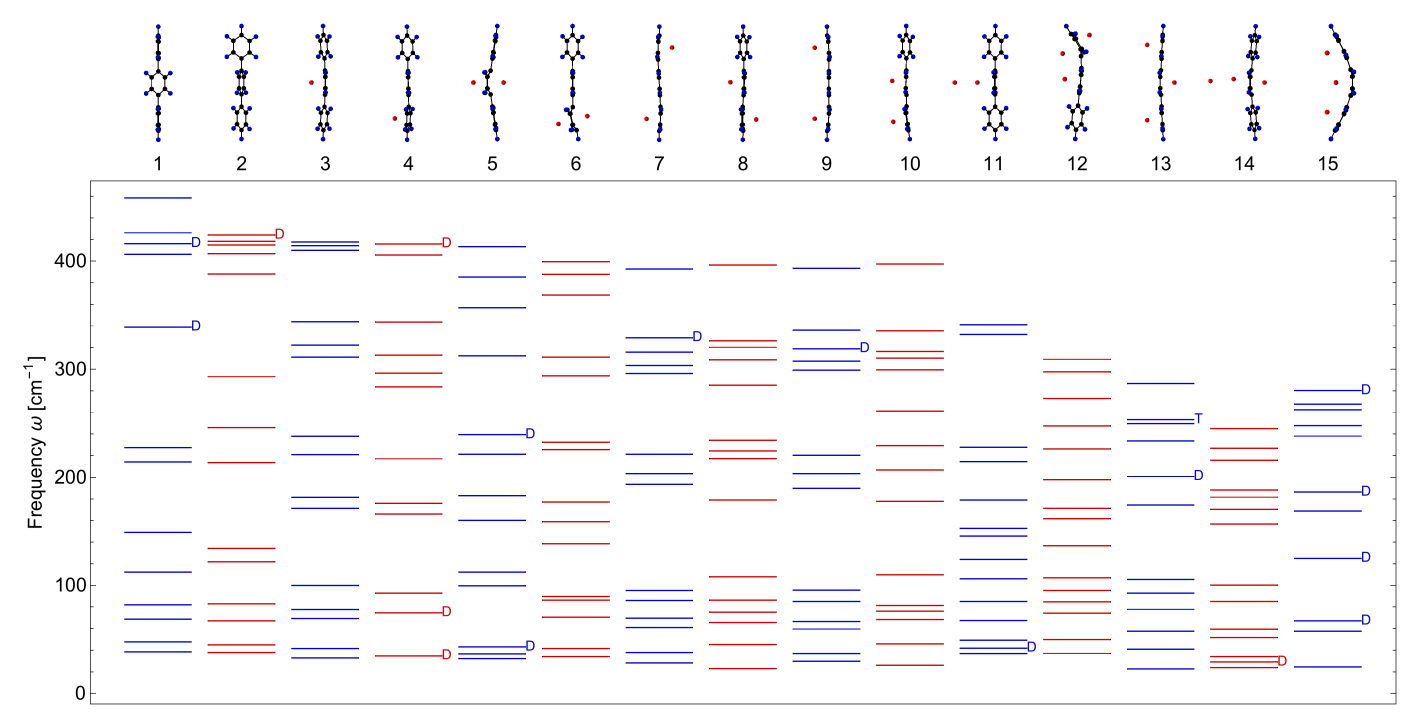

Figure 4: Vibrational spectra of triphenyl and triphenyl with Li adsorption.

$e$ resonating valence patterns to have the excess- $e$ delocalize between rings as would be facilitated by enhancing inter-ring coupling by having neighbor rings aligned in a common plane. Of course the effect of delocalization should fall off as the distance from the Li increases, so that for sufficient number of ring to Li ratio, the twisting of rings should again set in (at the rings a greater distance from $\mathrm{Li}$ ). Moreover for Li-doped anthracenes delocalization of the excess-e should tend to suppress the local distortion seen. The distinction of behavior between pentaphenyl and anthracenes comes depending on how the resonance is enhanced for the anthracene by localizing the defect in the center ring to leave 2 sextets in the terminal rings; whereas for pentaphenyl by delocalizing the excess-e defect (through all different rings, it being seen that localization does not in this case lead to any additional aromatic sextets) [22, 23].

To confirm the observation we show in Figure 5 in the first two columns heptaphenyl with one and two sided adsorption of $8 \mathrm{Li}$ and equivalent configurations for octaphenyl are in the two columns. Indeed the behavior is analogue to the one seen for tetraphenyl. We also attempted to calculate a configuration with $16 \mathrm{Li}$ adsorbed, eight on each side, but we found only irregular structures, as had already been the case for tetraphenyl. We may note however that structures with double adsorption to alternating rings are quite regular, though best applied to odd numbers of rings to avoid a asymmetry between the ends of the chains. In this case we have found a spontaneous symmetry breaking similar to what we have found for polyacenes resulting on a zigzag structure. It is shown in Figure 6 comparing nonacene and nonaphenyl where the inside angles are $160^{\circ}$ and $164.6^{\circ}$ respectively. In infinite (or very long chains) a Peierls transition [24] is almost certain to occur.

\section{Conclusions}

In this paper we present a theoretical, largely numerical study of adsorption of lithium to quasi-one dimensional conjugated

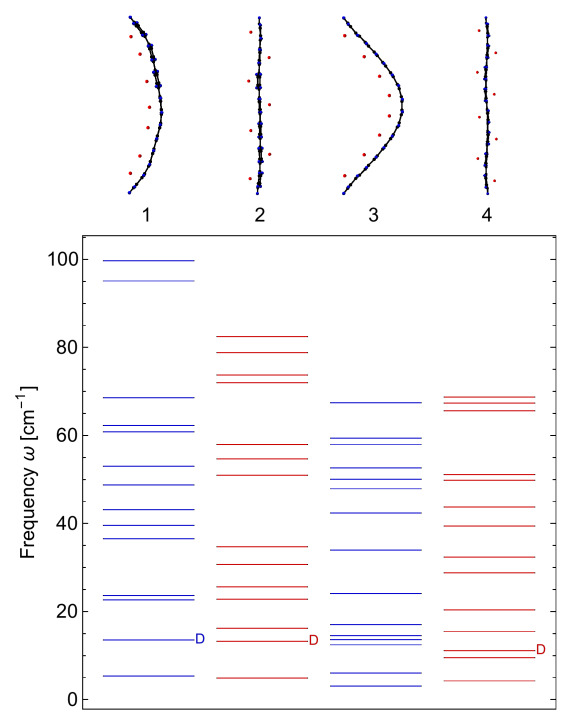

Figure 5: Vibrational modes of heptaphenyl (column 1 and 2) and octaphenyl under Li adsorption (column 3 and 4).

carbon structures, namely polyacenes and poly-p-phenylenes (PPPs). We analyze the vibrational spectra and qualitatively the movement of vibrational modes. We have found that different adsorbate configurations lead to significantly different vibrational spectra. The spontaneous symmetry breaking, which is observed for some adsorbate configurations, does not show a characteristic signature in the vibrational spectrum. Notably we find that the torsion in the PPPs is greatly diminished and possibly eliminated by the alkaline adsorbates (shown for lithium, but verified for other alkalines), if these are more or less regularly spaced. This aspect is of importance. Polyacenes are theoretically very appealing, as the qualitative behavior of the vibrational modes is similar. Yet as far as actual realizations are concerned PPPs have practical advantages because they are less reactive. Thus we have similar structures for the two types 
(a)

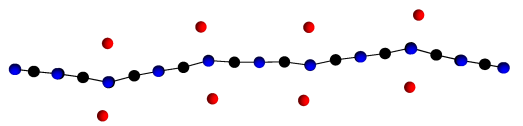

(b)

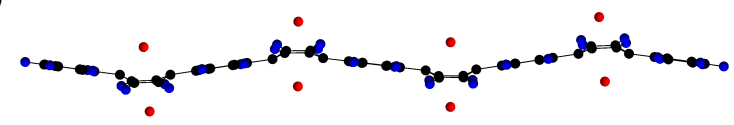

Figure 6: (a) Lateral view from adsorption of four pairs of lithium atoms alternating ring on nonacene, $(c)$ Lateral view from adsorption of four pairs of lithium atoms alternating ring on nonaphenyl

of molecules once they carry the corresponding adsorbates. We also speculate about possibilities to use the deposited atoms as qubits and two modes of vibrations, as extended qubits in order to mediate control gates.

\section{Acknowledgments}

Financial support from CONACyT research grant 219993 and PAPIIT-DGAPA-UNAM research grants IG100616 and IG101113 is acknowledged. We acknowledge extensive use of the MIZTLI super computing facility of DGTIC-UNAM.

\section{Appendix A. Supplementary material}

The supplementary material contains movie clips of the lowest three vibrational modes of the studied molecules. The movies are named as $n_{1}-n_{2}-n_{3}$, where $n_{1}$ is the number of the figure, $n_{2}$ is the number of the molecule indicated in the corresponding figure, and $n_{3}$ is the number of the mode. For example, the movie clip 1-2-3 is the third mode of the second molecule in Figure 1.

The movie clips can be found also at http://www.cicc.unam.mx/vibmod/v3.

\section{References}

\section{References}

[1] Y. Ortiz, T. Seligman, Spontaneous symmetry breaking by double lithium adsorption in polyacenes, AIP Conf. Proc. 1323 (2010) 257.

[2] Y. Ortiz, Consecuencias geometricas y dinamicas de la multiple adsorcion de litio y otros alcalineos en poliacenos, poli-para-fenilenos y hojuelas de grafeno, Ph.D. thesis, Universidad Nacional Autónoma de México (2014).

[3] A. Jalbout, Y. Ortiz, T. Seligman, Spontaneous symmetry breaking and strong deformations in metal adsorbed graphene sheets, Chemical Physics Letters 564 (0) (2013) $69-72$.

[4] Y. P. Ortiz, D. J. Klein, T. H. Seligman, Lithium adsorbtion on polyacenes \& zig-zag-edge graphene strips, https://arxiv.org/abs/ 1603.09308

[5] A. Jalbout, T. Seligman, Electron localization on molecular surfaces by metal adsorption, J. Comput. Theor. Nanosci. 6 (2009) 541.

[6] L. Brey, H. A. Fertig, Electronic states of graphene nanoribbons studied with the dirac equation, Phys. Rev. B 73 (2006) 235411. doi:10.1103/ PhysRevB.73.235411

[7] T. Stegmann, J. A. Franco-Villafañe, U. Kuhl, F. Mortessagne, T. H. Seligman, Transport gap engineering by contact geometry in graphene nanoribbons: Experimental and theoretical studies on artificial materials, Phys. Rev. B 95 (2017) 035413. doi:10.1103/PhysRevB.95.035413
[8] F. Schmidt-Kaler, H. Häffner, S. Gulde, M. Riebe, G. Lancaster, T. Deuschle, C. Becher, W. Hänsel, J. Eschner, C. Roos, R. Blatt, How to realize a universal quantum gate with trapped ions, Appl. Phys. B 77 (2003) 789

[9] H. Häffner, C. Roos, R. Blatt, Quantum computing with trapped ions, Phys. Rep. 469 (2008) 155.

[10] Y. Ortiz, D. Bhattacharya, D. J. Klein, J. F. Liebmand, Super-molecules, Rev. Roum. Chim. 61 (2016) 269-276.

[11] M. J. Frisch et al., GAUSSIAN 09 (Revision A.02), 2009.

[12] P. Hohenberg, W. Kohn, Inhomogeneous electron gas, Phys. Rev. 136 (1964) 864.

[13] A. D. Becke, Density-functional exchange-energy approximation with correct asymptotic behavior, Phys. Rev. A 38 (1988) 3098-3100.

[14] R. Ditchfield, W. J. Hehre, J. A. Pople, Self-consistent molecular-orbital methods. ix. an extended gaussian-type basis for molecular-orbital studies of organic molecules, J. Chem. Phys. 54 (2) (1971) 724-728. doi: 1

[15] J. Perdew, Electronic Structure of Solids '91, Akademie Verlag, Berlin, 1991, p. 11

[16] C. Adamo, V. Barone, Exchange functionals with improved long-range behavior and adiabatic connection methods without adjustable parameters: The mpw and mpw1pw models, J. Chem. Phys. 108 (2) (1998) 664 675. doi:10.1063/1.475428

[17] B. J. Lynch, Y. Zhao, D. G. Truhlar, Effectiveness of diffuse basis functions for calculating relative energies by density functional theory, J. Phys. Chem. A 107 (9) (2003) 1384-1388. doi:10.1021/jp0215901

[18] A. D. Becke, Density-functional thermochemistry. iii. the role of exact exchange, J. Chem. Phys. 98 (7) (1993) 5648-5652. doi:10.1063/1. 464913

[19] A. P. Scott, L. Radom, Harmonic vibrational frequencies: An evaluation of hartree-fock, moller-plesset, quadratic configuration interaction, density functional theory, and semiempirical scale factors, The Journal of Physical Chemistry 100 (41) (1996) 16502. doi:10.1021/jp960976r

[20] M. D. Halls, H. B. Schlegel, Comparison of the performance of local, gradient-corrected, and hybrid density functional models in predicting infrared intensities, The Journal of Chemical Physics 109 (24) (1998) 10587-10593. doi:10.1063/1.476518

[21] G. S.-M. Tong, A. S.-C. Cheung, Density functional theory study of alkali metal-noble metal diatomic molecules, The Journal of Physical Chemistry A 106 (47) (2002) 11637-11643. doi:10.1021/jp026550w

[22] D. J. Klein, A. T. Balaban, Clarology for conjugated carbon nanostructures: Molecules, polymers, graphene, defected graphene, fractal benzenoids, fullerenes, nano- tubes, nano-cones, nano-tori, etc., Open J. Org. Chem. 5 (2011) 27-61.

[23] E. Clar, The Aromatic Sextet, John Wiley \& Sons, 1972.

[24] R. Peierls, Quantum theory of solids, Oxford, 1955. 